\title{
SIMULTANEOUS APPROXIMATION IN THE DIRICHLET SPACE
}

\author{
ARNE STRAY
}

\section{Introduction}

Let $\mathscr{D}$ denote all analytic functions $f$ in the unit disc $\mathrm{D}$ such that the Dirichlet integral

$$
D(f)=\iint\left|f^{\prime}\right|^{2} d x d y<\infty
$$

is finite. We characterize the subsets $F$ of $D$ with the following property: Whenever $f \in \mathscr{D}$, there are polynomials $p_{n}, n=1,2, \ldots$ such that

$$
D\left(f-p_{n}\right) \rightarrow 0
$$

and

$$
\sup \left(\left|p_{n}(z)\right|, z \in F\right) \rightarrow \sup (|f(z)|, z \in F)
$$

as $n \rightarrow \infty$. We also characterize those sets $F \subset D$ with the property

$$
\sup (|f(z)|, z \in F)=\sup (|f(z)|, z \in D)
$$

for all $f \in \mathscr{D}$. The characterizations involve geometric properties of $F$ as well as certain concepts from potential theory. Let $T$ denote the unit circle. The set $\bar{F} \cap T$, consisting of all limit points of $F$ on $T$ is split into a disjoint union

$$
\bar{F} \cap T=F_{\mathrm{t}} \cup F_{\mathrm{nt}}
$$

where $F_{\mathrm{t}}$ and $F_{\mathrm{nt}}$ are called the tangential and non-tangential part of $\bar{F} \cap T$. A point $w$ belongs to $F_{\mathrm{nt}}$ if and only if there is $z_{n} \in F$ converging to $w$ such that $\left|w-z_{n}\right|\left(1-\left|z_{n}\right|\right)^{-1}$ remains bounded as $n \rightarrow \infty$. If $z \in D, z \neq 0$, let $I_{z}=\{w \in T:|w-z|<2(1-|z|)\}$. For $m=1,2, \ldots$, let

$$
S_{m}(F)=\cup I_{z}, \quad z \in F, \quad|z|>1-m^{-1}
$$

We shall identify $T$ with the interval $[-\pi, \pi)$ on the real line $\mathscr{R}$ when speaking about the capacity $\operatorname{cap}(B)$ or the linear measure $|B|$ of a subset $B$ of $T$. The 
capacity we use is the so called Bessel capacity $C^{\frac{1}{2}, 2}(B)$ as defined in [1], page 21 . We remark that this capacity is equivalent to logaritmic capacity. We can now formulate our main results:

THEOREM 1.1. If $F$ is a subset of the unit disc $D$, the following statements are equivalent

(a) If $\in \mathscr{D}$, there are polynomials $p_{n}, n=1,2, \ldots$, such that $D\left(f-p_{n}\right) \rightarrow$ 0 and $\sup \left(\left|p_{n}\right|, z \in F\right) \rightarrow \sup (|f(z)|, z \in F)$ as $n \rightarrow \infty$.

(b) For $m=1,2, \ldots, S_{m}(F) \cup F_{\mathrm{nt}}$ is thick at almost all $z \in \bar{F} \cap T$. The exceptional set has zero capacity.

(c) For $m=1,2, \ldots, S_{m}(F)$ is thick at allmost all $z \in \bar{F} \cap T$. The exceptional set has zero capacity.

The word "thick" in Theorem 1.1 should be understood in the sense of potential theory in connection with Wiener's criterion. See Section 2 for more details. Theorem 1.1 solves the problem of characterizing the Farrell sets for $\mathscr{D}$. This is part of a general problem in approximation theory raised by L. A. Rubel. See [16] and [18]. Results about Farrell sets for other function spaces can for example be found in [12], [11], [17] and [20].

THEOREM 1.2. Let $F$ be a subset of $D$. The following statements are equivalent

(a) For all $f \in \mathscr{D}$ the supremum of $|f|$ on $F$ equals the supremum of $|f|$ on $D$.

(b) $\operatorname{cap}(T)=\operatorname{cap}\left(T \backslash F_{\mathrm{t}}\right)$.

Condition (b) in Theorem 1.2 appeared in a theorem by L. V. Ahlfors and A. Beurling characterizing removable singularities for analytic functions with finite Dirichlet integral. See [8], page 82-85, for details. We use their theorem as well as Theorem 1.1 to deduce Theorem 1.2.

If $\mathscr{A}$ is any space of functions in $D$, a set $F \subset D$ is called a set of determination for $\mathscr{A}$ if

$$
\sup \{|f(z)|, z \in F\}=\sup \{|f(z)|, z \in D\}
$$

whenever $f \in \mathscr{A}$. Sets of determination are known for various function spaces. In particular we mention [5] (bounded harmonic functions), [13] (differences of positive harmonic functions) and [6], (bounded analytic functions). For extensions to higher dimensions see [10]. It is interesting to compare Theorem 1.2 with the result L. Brown, A. L. Shields and K. Zeller found: $F$ in a set of determination for $H^{\infty}(D)$ if and only if $\left|T \backslash F_{\mathrm{t}}\right|=|T|$. ( $H^{\infty}$ denotes the space of all bounded analytic functions in $D$ ). The rest of the paper is organized as follows: In Section 2 we give some background about $\mathscr{D}$ and potential theory. In Section 3 we state and prove a theorem relating Farrell sets to other 
approximation problems. Then Theorem 1.1 is proved in Section 4. We make extensive use of the Bessel potential space $L^{\frac{1}{2}, 2}(\mathscr{R})$. A convenient reference in this connection is the recent book [1]. In Section 5 we add some final remarks including a proof of Theorem 1.2.

\section{Potential theory and the space $\mathscr{D}$}

If $w \in T$ and $\alpha>1$, we define

$$
\Gamma_{\alpha}(w)=\{z \in D:|w-z| \leq \alpha(1-|z|)\}
$$

Lemma 2.1. If $f \in \mathscr{D}$, the limit $f(w)=\lim f(z), z \rightarrow w, z \in \Gamma_{\alpha}(w)$, exists for almost all $w \in T$. The exceptional set has zero capacity.

Proof. See [4] or [21] page 344. More general results can be found in [1] page 161 or [8] page 55 .

We shall need that the Dirichlet integral can be expressed as a boundary integral

$$
D(f)=\int_{-\pi}^{\pi} \int_{-\pi}^{\pi} \frac{|\tilde{f}(s-t)-\tilde{f}(s)|^{2}}{\left|e^{i t}-1\right|^{2}} d s d t
$$

where $\tilde{f}$ denotes the $2 \pi$ periodic function corresponding to $f$ by $\tilde{f}(t)=$ $f\left(e^{i t}\right), t \in \mathscr{R}$.

We also consider the Bessel potential space $L^{\frac{1}{2}, 2}(\mathscr{R})$ and recall that a function $g$ belongs to $L^{\frac{1}{2}, 2}(\mathscr{R})$ if and only if

$$
\int_{-\infty}^{\infty} \int_{-\infty}^{\infty} \frac{|g(x-y)-g(x)|^{2}}{y^{2}} d x d y
$$

is finite.

If $\phi \in C^{\infty}(\mathscr{R})$ has compact support, it follows directly from (2) that $\phi g \in L^{\frac{1}{2}, 2}(\mathscr{R})$ whenever $g \in L^{\frac{1}{2}, 2}(\mathscr{R})$. Our next result is certainly well known and easy to prove.

Lemma 2.2. Let $\phi \in C^{\infty}(\mathscr{R})$ have compact support on $(-\pi, \pi)$

(a) If $g$ is defined on $\mathscr{R}$ by $g=\tilde{f}$ for some $f \in \mathscr{D}$, then $\phi g \in L^{\frac{1}{2}, 2}(\mathscr{R})$.

(b) Let $h \in L^{\frac{1}{2}, 2}(\mathscr{R})$ be real-valued. Define $u=\phi g$ on $(-\pi, \pi)$. Then $u=\Re \tilde{f}$ for some $f \in \mathscr{D}$.

Proof. Since $\left|\frac{e^{i t}-1}{t}\right| \rightarrow 1$ as $t \rightarrow 0$, the integrals (1) and (2) are simultaneously bounded for $\phi g$. The same argument applies to $u=\phi h$ as defined in (b) and then Douglas formula (See [3]), gives

$$
\iint_{D}|\nabla u|^{2} d x d y<\infty
$$


where $u$ is extended to $D$ by Poissons formula. If $v$ is a harmonic conjugate to $u$ in $D$, the Cauchy-Riemann equations give $u+i v \in \mathscr{D}$.

The reason why we formulated Lemma 2.2 is that we wish to apply various results about $L^{\frac{1}{2}, 2}(\mathscr{R})$ as expressed in the recent book [1] to the Dirichlet space $\mathscr{D}$. The corresponding results for the Bessel potential space $L^{\frac{1}{2}, 2}(T)$ do not seem equally accessible through the existing literature.

If $B \subset \mathscr{R}$ and $x_{0} \in \mathscr{R}$, we say that $B$ is thin at $x_{0}$ if

$$
\int_{0}^{1} \frac{\operatorname{cap}\left(B \cap\left(x_{0}-r, x_{0}+r\right)\right)}{r} d r<\infty
$$

If the above integral diverges, we say that $B$ is thick at $x_{0}$. See [1] page 166 for more details.

We end this section by a remark about truncation of functions in $L^{\frac{1}{2}, 2}(\mathscr{R})$ and $\mathscr{D}$. If $u \in L^{\frac{1}{2}, 2}(\mathscr{R})$ is realvalued and $M$ is a real constant, the function

$$
u_{M}(x)=\min (u(x), M), \quad x \in \mathscr{R}
$$

is called a truncation of $u$. From (2) it is easy to see that $u_{M} \in L^{\frac{1}{2}, 2}(\mathscr{R})$. From (1) it follows that $\Re f$ may be truncated in the same way if $f \in \mathscr{D}$. Even functions in $\mathscr{D}$ may be truncated. For more details see [7].

\section{Farrell sets and other approximation problems}

We use the notation $\|g\|_{B}=\sup (|f(z)|, z \in B)$ if $g$ is a function defined on a set $B$. We also use the norm

$$
\|f\|=\left[|f(0)|^{2}+\iint_{D}\left|f^{\prime}\right|^{2} d x d y\right]^{\frac{1}{2}}, \quad f \in \mathscr{D}
$$

making $\mathscr{D}$ a Hilbert space.

The main result in this section is

THEOREM 3.1. The following statements are equivalent for a subset $F$ of $D$ :

(a) If $f \in \mathscr{D}$ there are polynomials $p_{n}$ such that $\left\|p_{n}-f\right\| \rightarrow 0$ and $\left\|p_{n}\right\|_{F} \rightarrow\|f\|_{F}$ as $n \rightarrow \infty$.

(b) If $f \in \mathscr{D}$ and $u$ is a uniformly continuous function on $F$, there are polynomials $p_{n}$ such that $\left\|p_{n}-f\right\| \rightarrow 0$ and $\left\|p_{n}-u\right\|_{F} \rightarrow\|f-u\|_{F}$ as $n \rightarrow \infty$.

Proof. We only need to prove (a) $\Rightarrow$ (b). We follow an idea that previously was used in a similar situation for the Hardy space $H^{p}(D), 0<p<\infty$. (See [12]). Our next lemma is useful in order to localize the approximation problem in Theorem 3.1. 
Lemma 3.2. Let $F$ be a Farrell set for $\mathscr{D}$. Suppose $f \in \mathscr{D}$ is bounded on $F$ and that $|f| \leq t$ on $F \cap \Delta$, for some disc $\Delta$ and some $t>0$. Then $\left|f\left(e^{i \theta}\right)\right| \leq t$ for almost all $e^{i \theta} \in \bar{F} \cap \Delta \cap T$. The exceptional set has zero capacity.

Proof. Let $z_{0} \in \bar{F} \cap \Delta \cap T$ and choose a smooth function $\phi$ such that $\phi=0$ near $z_{0}, \phi \leq 0$ on $T$, and define $f_{\phi}=f e^{\phi+i \tilde{\phi}}$ where $\tilde{\phi}$ is a harmonic conjugate to the (harmonic) extension of $\phi$ to $D$. We may choose $\phi$ such that $\left|f_{\phi}\right| \leq t$ on $F$ and $f_{\phi} \in \mathscr{D}$. If $\left\{q_{v}\right\}$ are polynomials such that $\left\|f_{\phi}-q_{v}\right\| \rightarrow 0$, it is well known that $\operatorname{cap}\left\{\theta:\left|f_{\phi}\left(e^{i \theta}\right)-q_{v}\left(e^{i \theta}\right)\right|>\epsilon\right\} \rightarrow 0$ as $v \rightarrow \infty$ for any $\epsilon>0$. See for example ([1], Prop 2.3.8). Hence if $\left\|q_{v}\right\|_{F} \rightarrow\left\|f_{\phi}\right\|_{F} \leq t$, it follows that $\left|f_{\phi}\right| \leq t$ on $\bar{F} \cap T$, except for a set of zero capacity. Since $\left|f_{\phi}\right|=|f|$ on $T$ near $z_{0}$, Lemma 3.2 follows.

Our next lemma is the key both to prove Theorem 3.1 and to obtain the geometric characterization in Theorem 1.1.

Lemma 3.3. Let $F$ be a subset of the unit disc D. The following statements are equivalent:

(a) $F$ is a Farrell set for $\mathscr{D}$.

(b) If $f \in \mathscr{D}$, then $|f| \leq\|f\|_{F}$ on $\bar{F} \cap T$ with the exception of a set of zero capacity.

(c) If $f \in \mathscr{D}$ is bounded on $F$ and $\Delta$ is an open disc, there are polynomials $\left\{q_{v}\right\}$ such that $\left\|f-q_{\nu}\right\| \rightarrow 0,\left\|q_{\nu}\right\|_{F} \rightarrow\|f\|_{F}$ and $\left\|q_{\nu}\right\|_{F \cap \Delta} \rightarrow\|f\|_{F \cap \Delta}$

Proof. By Lemma 3.2, we only have to prove (b) $\Rightarrow$ (c). Let $f \in \mathscr{D}$ be bounded on $F$ and assume $\|f\|=1$. Given $\epsilon>0$, we define

$$
P_{\epsilon}=\left\{p \in P:\|p\| \leq 1,\|p\|_{F} \leq\|f\|_{F}+\epsilon,\|p\|_{F \cap \Delta} \leq\|f\|_{F \cap \Delta}+\epsilon\right\} .
$$

Here $P$ denotes the set of polynomials. If $K \subset D$ is compact, we prove that $f$ is uniformly approximable on $K$ by functions from $P_{\epsilon}$. Let $\mu$ denote a measure on $K$ satisfying

$$
|\mu(p)| \leq 1, \quad p \in P_{\epsilon}
$$

The restrictions of functions in $P_{\epsilon}$ to the space $C(K)$ consisting of all continuous functions on $K$, is a convex set. By the separation theorem for convex sets and the Riesz representation theorem, it is sufficient to prove that $|\mu(f)| \leq 1$.

To obtain this we consider the Banach space

$$
L=\mathscr{D} \times C(\overline{F \backslash \Delta}) \times C(\overline{F \cap \Delta})
$$

with the norm

$$
N\{a, b, c\}=\max \left\{\|a\|,\left(t_{1}+\epsilon\right)^{-1}\|b\|_{\overline{F \backslash \Delta}},\left(t_{2}+\epsilon\right)^{-1}\|c\|_{\overline{F \cap \Delta}}\right\}
$$


where $t_{1}=\|f\|_{F}$ and $t_{2}=\|f\|_{F \cap \Delta}$. By the Hahn-Banach theorem the linear functional $p \rightarrow \mu(p)$ admits a normpreserving extension from $P$ to $L$. We represent this extension by a triple $\left(g, \mu_{1}, \mu_{2}\right)$ where $g \in \mathscr{D}$ (note that $\mathscr{D}$ is its own dual), and $\mu_{1}$ and $\mu_{2}$ are measures on $\overline{F \backslash \Delta}$ and $\overline{F \cap \Delta}$ respectively.

Since we are dealing with a normpreserving extension, we can write (with $\langle h, g\rangle=h(0) \overline{g(0)}+\iint_{D} h^{\prime} \overline{g^{\prime}} d x d y$ for $\left.f, g \in \mathscr{D}\right)$

$$
\mu\left(z^{n}\right)=\left\langle z^{n}, g\right\rangle+\mu_{1}\left(z^{n}\right)+\mu_{2}\left(z^{n}\right), \quad n \geq 0
$$

and

$$
\|g\|+\left(t_{1}+\epsilon\right)\left|\mu_{1}\right|+\left(t_{2}+\epsilon\right)\left|\mu_{2}\right| \leq 1
$$

where $|\lambda|$ denotes the total variation of the measure $\lambda$.

By (4) and a "restoration theorem" of S. Hruscev ([15], page 440), it follows that $\mu_{1}(B)=\mu_{2}(B)=0$ if $B \subset T$ has zero capacity. Actually we can not apply Hruscev's theorem directly since it deals with measures supported on $T$. But if we define measures $\lambda_{1}$ and $\lambda_{2}$ on $T$ by

$$
\lambda_{i}(\phi)=\iint_{D} \phi d \mu_{i}, \quad i=1,2 .
$$

for all continuous $\phi$ on $\bar{D}$ being harmonic in $D$, then $\lambda_{1}$ and $\lambda_{2}$ are uniquely defined measures on $T$ being absolutely continuous with respect to linear measure on $T$. The desired conclusion about $\mu_{1}$ and $\mu_{2}$ follows by applying Hruscev's theorem to $\lambda$ given by

$$
\lambda=\sum_{i=1}^{2} \lambda_{i}(B)+\mu_{i}(B)
$$

if $B$ is a Borel set contained in $T$.

To complete the proof, let us first assume $f$ is bounded on $D$. Let $f_{r} \in \mathscr{D}$ be defined by $f_{r}(z)=f(r z), z \in D, 0<r<1$. Since the Taylor series of $f_{r}$ converge uniformly to $f_{r}$ near $\bar{D}$, we conclude that

$$
\mu\left(f_{r}\right)=\left\langle f_{r}, g\right\rangle+\mu_{1}\left(f_{r}\right)+\mu_{2}\left(f_{r}\right)
$$

for all $r, 0<r<1$. Letting $r \rightarrow 1$ we get by dominated convergence that

$$
\mu(f)=\langle f, g\rangle+\mu_{1}(f)+\mu_{2}(f)
$$

By combining the hypothesis (b) in Lemma 3.3 and the inequality (5), we conclude that $|\mu(f)| \leq 1$. 
Since $\epsilon$ and $K$ were arbitrary, we can so far conclude that there are polynomials $q_{v}$ converging weakly to $f$ in $\mathscr{D}$ and satisfying the two last requirements in (c) in Lemma 3.3. By taking convex combinations of polynomials from $\left\{q_{v}\right\}$ we get (c) in Lemma 3.3 provided $f$ is bounded. By a representation formula for the Dirichlet integral due to L. Carleson [7], any $f$ in $\mathscr{D}$ may be approximated pointwise in $D$ by bounded functions $f_{n} \in D$ such that $\left\|f_{n}\right\|$ is bounded and $\left|f_{n}(z)\right| \leq|f(z)|$ for $z \in D$. Hence (c) follows and Lemma 3.3 is proved.

We now prove (a) $\Rightarrow$ (b) in Theorem 3.1 by a constructive argument. Let $\phi$ denote a smooth function with compact support in the complex plane $\mathscr{C}$. If $h$ is locally integrable on $\mathscr{C}$, we recall the definition of the $T_{\phi}$-operator (See [9], page 210)

$$
T_{\phi} h(w)=\phi(w)+\frac{1}{\pi} \iint \frac{h(z)}{z-w} \frac{\partial \phi}{\partial \bar{z}} d x d y(z)
$$

If $h$ belongs to $L^{p}(d x d y)$ locally, $p>2$, we have

$$
\frac{\partial}{\partial \bar{w}} T_{\phi} h=\phi \frac{\partial h}{\partial \bar{w}}
$$

and

$$
\frac{\partial}{\partial w} T_{\phi} h=\frac{\partial}{\partial w}(\phi h)+T h
$$

where

$$
T h(w)=\frac{-1}{\pi} \iint \frac{h(z)}{(z-w)^{2}} d x d y(z)
$$

denotes the planar Hilbert transform. We refer to [2], page 85-90 and [9] for more details.

Let us now consider $f \in \mathscr{D}$ being bounded on $F$ and $g$ uniformly continuous on $F$. For the moment we assume $f$ admits analytic continuation across $T \backslash \bar{F}$. Given $\epsilon>0$, we cover $\bar{F}$ by a finite collection of open discs $\left\{\Delta_{j}\right\}$ such that $|g(z)-g(w)|<\epsilon$ if $z, w \in \Delta_{j} \cap F$ for some $j$. We select constants $g_{j}=g\left(z_{j}\right)$ where $z_{j} \in \Delta_{j} \cap F$.

By Lemma 3.2 and Lemma 3.3, we find for each $j$ polynomials $q_{v}^{j}$ such that

$$
\left\|q_{v}^{j}-\left(f-g_{j}\right)\right\| \rightarrow 0
$$

and

$$
\left\|q_{\nu}^{j}\right\|_{F \cap \Delta_{j}} \rightarrow\left\|f-g_{j}\right\|_{F \cap \Delta_{j}}
$$

and such that $\left\|q_{v}^{j}\right\|_{F}$ is bounded independent of $v$ and $j$. 
Since $f$ is analytic near $\bar{D} \backslash \cup \Delta_{j}$, there are polynomials $\underline{p}_{v}$ approximating $f$ uniformly near $\bar{D} \backslash \cup \Delta_{j}$. Let $q_{v}$ be given by $q_{v}=p_{v}$ on $\bar{D} \backslash \cup \Delta_{j}$, and

$$
q_{v}(z)=q_{\nu}^{j}(z)+g_{j}, \quad z \in \Delta_{v} \backslash \cup_{1}^{\nu-1} \Delta_{k}
$$

for $v=1,2, \ldots, j$. These "P-splines" $q_{v}$ converge to $f$ in the sense that

$$
\iint_{D}\left|f^{\prime}-q_{\nu}^{\prime}\right|^{2} d x d y \rightarrow 0
$$

as $v \rightarrow \infty$. Moreover, it is easy to verify that

$$
\left\|q_{v}-g\right\|_{F} \leq\|f-g\|_{F}+2 \epsilon
$$

for $v$ sufficiently large.

The only problem with $\left\{q_{v}\right\}$ is the discontinuity near $E=\cup \partial \Delta_{j} \cap\{z$ : $|z| \leq 1\}$. We modify $q_{\nu}$ near $E$ using the $T_{\phi}$-operator introduced above.

Let $\left\{D_{k}\right\}$ denote a finite covering of $E$ by open discs $D_{k}$ and let $\left\{\phi_{k}\right\}$ denote smooth functions supported in $D_{k}$ such that

$$
\sum \phi_{k}=1
$$

near $E$. We assume $D_{k} \cap T=\emptyset$ if $1 \leq k \leq k_{0}$ and if $k>k_{0}$ we assume $D_{k}$ is centered at $\zeta_{k} \in T$ and that the nontangential limit

$$
\lambda_{k}=\lim f(z), \quad z \rightarrow \zeta_{k}, \quad z \in \Gamma_{\alpha}\left(\zeta_{k}\right)
$$

exists for some large $\alpha$ to be specified. This is possible to obtain by Lemma 2.1. We define

$$
\tilde{q_{v}}=q_{v}-\sum_{k=1}^{k_{0}} T_{\phi_{k}}\left(q_{v}-f\right)-\sum_{k>k_{0}} T_{\phi_{k}}\left(\left(q_{v}-\lambda_{k}\right) \chi_{\delta}\right)
$$

where $\chi_{\delta}$ is the characteristic function of $\{z:|z|<1+\delta\}$ and $\delta=\delta(v)$ is a small number to be specified.

Claim 1. $\tilde{q}_{v}$ is analytic near $\bar{D}$.

Proof. By (6) we have

$$
\frac{\partial}{\partial \bar{w}} \tilde{q}_{v}=\frac{\partial}{\partial \bar{w}} q_{v}\left(1-\sum_{k} \phi_{k}\right)=0
$$

near $\bar{D}$. 
Claim 2. $\left\|\tilde{q}_{v}-g\right\|_{F} \leq\|f-g\|_{F}+3 \epsilon$.

Proof. Recall that $D_{k}$ is centered at $\zeta_{k} \in T$ if $k>k_{0}$. We assume that either $\zeta_{k} \in T \backslash \bar{F}$ or that $\zeta_{k}$ is an interior point relative to $\bar{F} \cap T$. When estimating $\left\|\tilde{q}_{v}-g\right\|_{F}$, the essential contribution comes from

$$
\left\|q_{v}-\sum_{k>k_{0}} \phi_{k}\left(q_{v}-\lambda_{k}\right)-g\right\|_{F}
$$

The terms we have neglected in doing this approximation are

$$
\sum_{1 \leq k \leq k_{0}} T_{\phi}\left(q_{v}-f\right)
$$

and

$$
\sum_{k>k_{0}} \iint \frac{\left(q_{v}-\lambda_{k}\right)}{(z-w)} \frac{\partial \phi}{\partial \bar{z}} \chi_{\delta} d x d y(z)
$$

The first of these sums is small when $v$ is large since $q_{v} \rightarrow f$ uniformly on compact subsets of $D$. The second sum is uniformly small in $w$ if $\alpha$ is large and $\delta$ is small, and the discs $D_{k}$ have small radii for $k>k_{0}$. We proceed to estimate $\left\|q_{v}-\sum_{k>k_{0}} \phi_{k}\left(q_{v}-\lambda_{k}\right)-g\right\|_{F}$ :

We may assume that the discs $D_{k}$ are pairwise disjoint and that $D_{k} \cap \bar{F}=\emptyset$ if the center $\zeta_{k} \notin \bar{F}$. By (9) we only have to estimate $\left\|q_{v}-\phi_{k}\left(q_{v}-\lambda_{k}\right)-g\right\|_{F \cap D_{k}}$.

But

$$
\begin{aligned}
\left\|q_{v}-\phi_{k}\left(q_{v}-\lambda_{k}\right)-g\right\|_{F \cap D_{k}} & \leq\left\|\left(q_{v}-g\right)\left(1-\phi_{k}\right)+\phi_{k}\left(\lambda_{k}-g\right)\right\|_{F \cap D_{k}} \\
& \leq \max \left\{\left\|q_{v}-g\right\|_{F \cap D_{k}},\left\|\lambda_{k}-g\right\|_{F \cap D_{k}} \|\right\} \leq\|f-g\|_{F}+2 \epsilon
\end{aligned}
$$

provided the radius $d_{k}$ of $D_{k}$ is sufficiently small. (To get the last inequality, it is essential that $\zeta_{k}$ is interior relative to $\left.\bar{F} \cap T\right)$.

Claim 3. $\left\|\tilde{q}_{v}-f\right\| \leq \epsilon$ provided $\left\{D_{k}\right\}$ are chosen properly and $v$ is sufficiently large.

Proof. We first remark that the planar Hilbert transform is an isometry on $L^{2}(\mathscr{C})$ ([2], page 89). From (6) and (7) it follows that the $T_{\phi}$-operator maps $\mathscr{D}$ into itself. By (8) we need only estimate

$$
\iint_{D \backslash E}\left|\tilde{q}_{v}^{\prime}-q_{\nu}^{\prime}\right|^{2} d x d y
$$

which equals

$$
\iint_{D \backslash E}\left|\sum_{k=1}^{k_{0}}\left\{T_{\phi_{k}}\left(q_{v}-f\right)-\sum_{k>k_{0}} T_{\phi_{k}}\left(\left(q_{v}-\lambda_{k}\right) \chi_{\delta}\right)\right\}^{\prime}\right|^{2} d x d y
$$


The first sum in (10) is easily estimated since $q_{v} \rightarrow f$ and $q_{v}^{\prime} \rightarrow f^{\prime}$ uniformly on the support of $\phi_{k}$ for $1 \leq k \leq k_{0}$.

The second sum in (10) is easily estimated using (6), (7) and the isometric property of the planar Hilbert transform. By construction we may choose $D_{k}$, $k>k_{0}$, so small that $\left\|q_{\nu}\right\|_{D \cap D_{k}}$ is bounded independently of $k$ and $v$. Using this bound and (8) and that $\delta=\delta(v)$ may be chosen as small as we wish, the second sum in (10) gives a small contribution provided the area of $\cup_{k>k_{0}} D_{k}$ is sufficiently small.

In the proof we assumed $f$ to admit analytic continuation across $T \backslash \bar{F}$. If $f_{1} \in \mathscr{D}$ is arbitrary it is sufficient to find $f$ as above such that

$$
\left\|f-f_{1}\right\|+\left\|f-f_{1}\right\|_{F}
$$

is as small as we please. This is easy to obtain since the $T_{\phi}$-operator maps $\mathscr{D}$ into itself. Indeed put $f_{1}=0$ outside $D$ and define

$$
f=f_{1}-\sum_{j} T_{\psi_{j}}\left(f_{1}\right)-R_{j}
$$

Here $\psi_{j}$ ars smooth functions with compact support disjoint from $\bar{F}$ such that $\sum_{j} \psi_{j} \equiv$ near $T \backslash \bar{F}$. We may assume $\psi_{j}$ is supported on a disc $K_{j}$ and that no more than $M$ of these discs overlap, where $M$ is a numerical constant. Moreover, if $h_{j}=T_{\psi_{j}} f_{1}$ then $R_{j}$ denotes the map in $\mathscr{D}$ given by

$$
R_{j}(z)=h_{j}\left(r_{j} z\right)
$$

for some $r_{j}<1$. We may choose $\left\{r_{j}\right\}$ such that

$$
\left\|h_{j}-R_{j}\right\|+\left\|h_{j}-R_{j}\right\|_{F} \leq \epsilon 2^{-j}
$$

for $j=1,2, \ldots$ The basic property (6) now gives that our function $f$ admits analytic continuation across $T \backslash \bar{F}$ and satisfies

$$
\left\|f_{1}-f\right\|+\left\|f_{1}-f\right\|_{F} \leq \epsilon
$$

For an alternative method to find $f \in \mathscr{D}$ approximating $f_{1}$ as above we refer to the proof of Lemma 3.2 in [19].

\section{Proof of Theorem 1.1}

In the proof of Theorem 1.1 we find it convenient to introduce another concept from potential theory: If $B$ and $S$ are Borel sets in $\mathscr{R}$ and $B \subset S$, we say that $B$ is representative for $S$ if $\operatorname{cap}(B \cap \Delta)=\operatorname{cap}(S \cap \Delta)$ for any disc $\Delta$. We refer to Theorem 11.4.2 and Remark 11.4 on page 327 in [1] for more about these 
sets. By Theorem 11.4.2 in [1], condition (c) in Theorem 1.1 can be restated as: $S_{m}(F)$ is representative for $S_{m}(F) \cup \bar{F} \cap T$ for any $m \geq 1$.

To prove (a) $\Rightarrow$ (c), we assume (c) fails while (a) holds. Then $L=S_{m}(F)$ fails to be representative for $L_{1}=S_{m}(F) \cup \bar{F} \cap T$ for some $m$. By Theorem 11.4.2 in [1] there is a disc $\Delta$ such that cap $\Delta \cap L<$ cap $\Delta \cap L_{1}$. By the definition of Bessel capacity there is $u \in L^{\frac{1}{2}, 2}(\mathscr{R})$ such that $u \geq 1$ on $L \cap \Delta$ and $u \leq t<1$ on a set $K \subset\left(L_{1} \backslash L\right) \cap \Delta$ having positive capacity. We choose $\phi \in C_{0}^{\infty}(\Delta)$ such that $0 \leq \phi \leq 1$ and define $v$ by $v=\phi(1-u)$. Then $v \geq 1-t$ on a set $K_{1} \subset K$ with cap $K_{1}>0$. Replacing $v$ by $\min \{v, 1-t\}$ if necessary, we may assume $v \leq(1-t)$ everywhere and $v \leq 0$ on $S_{m}(F)$. By Lemma 2.2, $w$ defined by

$$
w\left(e^{i \theta}\right)=v(\theta), \quad-\pi \leq \theta<\pi
$$

extends by Poisson's integral formula to a harmonic function in $D$ having finite Dirichlet integral. It is evident that $\|w\|_{F}<1-t$ since if $z \in F,|z|>1-\frac{1}{m}$, the contribution to $w(z)$ coming from integrating the Poisson kernel over $S_{m}(F)$ is significant.

If $\tilde{w}$ is a harmonic conjugate to $w$ in $D$ and $f=e^{w+i \tilde{w}}$, then $f \in \mathscr{D}$, $\|f\|_{F}<e^{1-t}$, while $|f| \geq e^{1-t}$ on a set $K_{1} \subset \bar{F} \cap T$ having positive capacity. By Lemma 3.3 this contradicts $F$ being a Farrell set and (a) $\Rightarrow$ (c) is proved. That $(\mathrm{c}) \Rightarrow$ (b) is trivial.

To prove (b) $\Rightarrow$ (a), we assume (b) holds while (a) fails. Again by Lemma 3.3 there is $f \in \mathscr{D}$ with $\|f\|_{F}<1$ and $|f| \geq 1$ on a set $B \subset \bar{F} \cap T$ with $\operatorname{cap}(B)>0$. By truncating $f$ if necessary (See [7]), we may assume $\|f\|_{D}=1$. Given $\epsilon>0$, we may even assume $\|f\|_{F}<\epsilon$ by replacing $f$ by $f^{N}$ for some integer $N$.

If $u=\Re\left(\frac{1}{2}\left(1+e^{i \alpha} f\right)\right)$, we can choose $\alpha$ real such that $u \geq 1-\frac{\epsilon}{2}$ on a subset $B^{\prime}$ of $B$ with $\operatorname{cap}\left(B^{\prime}\right)>0$. We also have $\|u\|_{F}<\frac{1}{2}(1+\epsilon)$ and $0 \leq u \leq 1$ on $[-\pi, \pi)$.

Recall that by assumption (b) the set $S_{m}(F) \cup F_{\text {nt }}$ is representative for $S_{m}(F) \cup \bar{F} \cap T$ for any $m \geq 1$. For each $m$ there is a subset $D_{m} \subset F_{\mathrm{t}}$ with cap $D_{m}=0$, such that $S_{m}(F) \cup F_{\text {nt }}$ is thick at $z_{0}$ for any $z_{0} \in F_{\mathrm{t}} \backslash D_{m}$. Let

$$
D_{0}=\cup_{1}^{\infty} D_{m}
$$

Then $\operatorname{cap}\left(D_{0}\right)=0$ and any set $S_{m}(F) \cup F_{\text {nt }}$ is thick at any $z_{0} \in F_{\mathrm{t}} \backslash D_{0}$.

Given such $z_{0}$, we may choose $m$ so large that if $I_{z} \cap B_{r}\left(z_{0}\right) \neq \emptyset$, then $I_{z} \subset$ $B_{2 r}\left(z_{0}\right)$ whenever $z \in F$ and $|z|>1-\frac{1}{m}$. (Here $B_{t}(z)=\{w:|w-z|<t\}$ )

We fix such an integer $m$ and shall use the function $u$ constructed above to get a contradiction at our point $z_{0} \in F_{\mathrm{t}} \backslash D_{0}$. Since $u(z)<\frac{1}{2}(1+\epsilon)$ if $I_{z} \in S_{m}(F)$, it follows by elementary estimates for the Poisson integral that 
$u \leq(1-\epsilon)$ on a set $E_{z} \subset I_{z}$ with $\left|E_{z}\right| \geq d\left|I_{z}\right|$ and $d$ is independent of $z$. Since $u$ is finely continuous outside a subset of $T$ having zero capacity ([1], page 177), we assume in the following that $u$ is finely continuous at $z_{0}$.

Let

$$
E=\bigcup_{z \in F,|z|>1-\frac{1}{m}} E_{z}
$$

CLAIM 4. There is a subset $E^{\prime}$ of $E$ such that $E^{\prime} \cup F_{\mathrm{nt}}$ is thick at $z_{0}$.

Taking the claim for granted and using that $u \leq(1-\epsilon)$ on $E^{\prime} \cup F_{\mathrm{nt}}$, it follows by fine continuity that $u\left(z_{0}\right) \leq(1-\epsilon)$. But this contradicts that $u \geq\left(1-\frac{\epsilon}{2}\right)$ on a set $B^{\prime} \subset F_{\mathrm{t}}$ of positive capacity.

It remains to prove the claim. With $m$ fixed as above, let $\left\{J_{v}\right\}$ denote a countable subcollection of $\left\{I_{z}: z \in F,|z|>1-\frac{1}{m}\right\}$ such that

$$
\bigcup_{v} J_{v}=\bigcup_{z \in F,|z|>1-\frac{1}{m}} I_{z}
$$

Also let $E_{v} \subset J_{v}$ be the corresponding subset with $\left|E_{v}\right| \geq d\left|J_{v}\right|$ and define

$$
E^{\prime}=\bigcup_{v} E_{v}
$$

Since $S_{m}(F) \cup F_{\text {nt }}$ is thick at $z_{0}$, the above claim follows from Wieners criterion (See [1], page 166) if we can show that

$$
\operatorname{cap}\left(\left(E^{\prime} \cup F_{\text {nt }}\right) \cap B_{2 r}\left(z_{0}\right)\right) \geq C_{0} \operatorname{cap}\left(\left(S_{m}(F) \cup F_{\text {nt }}\right) \cap B_{r}\left(z_{0}\right)\right)
$$

for all sufficiently small $r$ and with $C_{0}>0$ independent of $r$. By regularity properties of capacity ([1], page 28), it is sufficient to prove (11) if $S_{m}(F)$ is replaced by an arbitrary finite union $J=\cup_{1}^{N} J_{v}$ and $E^{\prime}$ is replaced by $\cup_{1}^{N} E_{v}$. By subadditivity of capacity we may even replace $\left\{J_{v}\right\}_{v=1}^{N}$ by a subcollection of pairwise disjoint intervals.

The following lemma immediately gives (11) and completes the proof of Theorem 1.1. (Recall that $m$ was large enough to guarantee $I_{v} \subset B_{2 r}\left(z_{0}\right)$ if $\left.I_{v} \cap B_{r}\left(z_{0}\right) \neq \emptyset\right)$

Lemma 4.1. Let $\left\{J_{v}\right\}$ be a finite collection of disjoint intervals and let for each $v, K_{v}$ be a subset of $J_{v}$ such that $\left|K_{v}\right| \geq d\left|J_{v}\right|$ with $d>0$. Then $\operatorname{cap}\left(\cup_{v} K_{v}\right) \geq C(d) \operatorname{cap}\left(\cup_{v} J_{v}\right)$ where $C(d)$ depends only on $d$.

The proof of Lemma 4.1 will not be given in detail since it is an immediate consequence of the definition of $C_{\frac{1}{2}, 2}$-capacity and the lower bounds for the capacitary potential $V_{\alpha, p}^{v}$ given in Lemma 9.8.3 in [1]. The set $F$ in Lemma 9.8.3 
corresponds to our set $\cup_{v} K_{v}$ and the measure " $v$ " in Lemma 9.8.3 is the capacitary measure for $F$.(see [1], page 21 for more about these concepts). That Lemma 9.8.3 applies in our situation follows since

$$
\operatorname{cap}\left(K_{v}\right) \geq c\left(\log \frac{2}{\left|K_{v}\right|}\right)
$$

for some constant $c$ and any $K_{v} \subset(-\pi, \pi]$.

\section{Concluding remarks}

We indicate the proof of Theorem 1.2: If $\bar{F} \supset T$, we have $S_{m}(F) \cup F_{\text {nt }}=T \backslash F_{m}$ where $F_{m}=F_{\mathrm{t}} \backslash S_{m}(F)$. By Theorem 1.1 and Theorem 13 in [14], we only have to prove

$$
\left\{\operatorname{cap} T=\operatorname{cap}\left(T \backslash F_{m}\right), m=1,2, \ldots\right\} \Rightarrow \operatorname{cap} T=\operatorname{cap}\left(T \backslash F_{\mathrm{t}}\right)
$$

So we assume cap $T=\operatorname{cap}\left(T \backslash F_{m}\right), m=1,2, \ldots$ and that

$$
\text { cap } T>\operatorname{cap}(T \backslash K)
$$

for some compact subset $K$ of $F_{\mathrm{t}}$. Then there is an analytic function $h_{K}$ in $\mathscr{C} \backslash K$ having finite Dirichlet integral in $\mathscr{C} \backslash K$ and being non constant. In fact, a theorem by L. V. Ahlfors and A. Beurling tells that (12) is necessary and sufficient for the existence of such a function $h_{K}$. (See [8], page 82-84).

In the following we assume $K$ is minimal in the sense that $h_{K}$ doesn't extend to be analytic near any $z \in K$. This minimal set is still denoted by $K$ and (12) will hold because of the Ahlfors-Beurling theorem. Let $K_{m}=K \cap F_{m}$. Then $K_{m}$ is compact and cap $T=\operatorname{cap}\left(T \backslash K_{m}\right)$ for all $m$. Let $z \in K_{m}$ and assume $\Delta$ is a disc centered at $z$ such that $\Delta \cap K \subset K_{m}$. If $\phi$ is a smooth function with compact support in $\Delta$ and $\phi \equiv 1$ near $z$, we define

$$
h_{\Delta}=T_{\phi}\left(h_{K}\right)
$$

By the Ahlfors-Beurling theorem and property (6) of the $T_{\phi}$-operator, $h_{\Delta}=0$, but on the other hand $h_{K}-h_{\Delta}$ is analytic near $z$ and this contradicts the assumed minimality of $K$.

We may therefore conclude that $K \backslash K_{m}$ is dense in $K$ for all $m$. By the Baire category theorem, the intersection of all $K \backslash K_{m}$ is dense in $K$, but on the other hand

$$
\bigcap_{m=1}^{\infty} K \backslash K_{m}=\emptyset
$$


We conclude that our initial assumption cap $T>\operatorname{cap}(T \backslash K)$ was false and this proves Theorem 1.2.

We finally show that in general the $m$-condition in Theorem 1.1 involving $S_{m}(F)$ cannot be removed. At the same time we get an example of a Farrell set for $\mathscr{D}$ where $\left|F_{\mathrm{t}}\right|>0$. We start with a compact totally disconnected subset $K$ of $T$ such that $|K|>0$ and cap $T=\operatorname{cap}(T \backslash K)$. Such sets exists (see [1], Remark 2, on page 314). Let

$$
T \backslash K=\bigcup I_{v}
$$

where the open $\operatorname{arcs} I_{v}$ are disjoint. We push each $I_{v}$ slightly into $D$ and obtain an $\operatorname{arc} \Lambda_{v}, v=1,2, \ldots$ Now let

$$
F=\bigcup_{v} \Lambda_{v}
$$

We can choose $\Lambda_{v}$ such that $F_{\mathrm{t}}=K$ while $F_{\mathrm{nt}}=\emptyset$. Since cap $T=$ $\operatorname{cap}(T \backslash K)$ it follows that $T \backslash K$ is thick at almost all $z \in K$ with respect to capacity (see [1], Theorem 11.4.2 combined with [14], Theorem 13). But we can choose the sets $\Lambda_{v}$ so close to $T$ that $S_{m}(F)=\left(\bigcup_{v} \Lambda_{v}\right) \backslash L_{m}, m=1,2, \ldots$ for some compact set $L_{m}$ of $\left.\bigcup_{v} \Lambda_{v}\right)$. This means that $S_{m}(F)$ is thick at almost all $z \in \bar{F}$ and $F$ is a Farrell set for $\mathscr{D}$ by Theorem 1.1 .

Let us finally remark that $S_{m}(f)$ could be replaced by

$$
S_{m}^{k}(F)=\left\{w:|w-z|<k(1-|z|) \text { for some } z \in F,|z| \geq 1-m^{-1}\right\}
$$

in Theorem 1.1 for any $k>1$, and the theorem would still remain true. This follows easily from Lemma 4.1.

\section{REFERENCES}

1. Adams, David R., and Hedberg, Lars Inge, Function Spaces and Potential Theory, Springer Verlag, 1996.

2. Ahlfors, Lars V., Lectures on Quasiconformal Mappings, van Nostrand Company, 1966.

3. Ahlfors, Lars V., Conformal Invariants, McGraw-Hill, New York, 1973.

4. Beurling, Arne, Ensembles exceptionelles, Acta Math, 72 (1940), 1-13.

5. Bonsall, F. F., Domination of the supremum of a bounded harmonic function by its supremum over a countable subset, Proc. Edinburgh Math. Soc. 32 (1987), 471-477.

6. Brown, Leon, Shields, Allen L., and Zeller, Karl, On absolutely convergent exponential sums, Trans. Amer. Math. Soc. 96 (1960), 162-183.

7. Carleson, Lennart, A representation formula for the Dirichlet integral, Math. Z. 56 (1960), 190-196.

8. Carleson, Lennart, Selected Problems on Exeptional Sets, Van Nostrand, Princeton, New Jersey, 1967.

9. Gamelin, T. W., Uniform Algebras, Prentice Hall, Englewood Cliffs, New Jersey, 1969. 
10. Gardiner, Stephen J., Sets of determination for harmonic functions, Trans. Amer. Math. Soc. 338 (1993), 233-243.

11. Gonzalez, Fernando P., and Gonzalez, Rodrigo T., Farrell and Mergelyan Sets for the Space of Bounded Harminic Functions, in Classical and Modern Potential Theory and Applications (Kluwer Academic Publishers, ed.), 1993.

12. Gonzales, Fernando P., and Stray, Arne, Farrell and Mergelyan sets for $H^{p}$ spaces, Michigan Math. J. 36 (1989), 379-386.

13. Hayman, Walter K., and Lyons, Terry J., Bases for positive continuous functions, J. London Math. Soc. 42 (1990), 292-308.

14. Hedberg, Lars I., Removable singularities and condenser capacities, Ark. Mat. 12 (1974), 181-201.

15. Nikolskii, N. K., Treatise on the Shift Operator, Springer Verlag, 1986.

16. Rubel, Lee A., A collection of research problems in mathematical analysis, Seminaire d'Analyse Moderne, Universite de Sherbrooke (1974).

17. Rubel, Lee A., Shields, Allen L., and Taylor, Brian A., Mergelyan sets and the modulus of continuity, in Approximation Theory, Academic Press, New York, 1973.

18. Rubel, Lee A., and Stray, Arne, Joint approximation in the unit disc, J. Approx. Theory 37 (1983), 44-50.

19. Stray, Arne, Approximation and interpolation for some spaces of analytic functions in the unit disc, Pacific J. Math. 54 (1974), 237-251.

20. Stray, Arne, Characterization of Mergelyan sets, Proc. Amer. Math. Soc. 44 (1974), 347-352.

21. Tsuji, M., Potential Theory in Modern Function Theory, Maruzen, Tokyo, 1959.

MATEMATISK INSTITUTT

UNIVERSITET I BERGEN

JOHS. BRUNSGT. 12

N-5008 BERGEN

NORWAY 